\title{
Thermoelectric and optical probes for a Fermi surface topology change in noncentrosymmetric metals
}

\author{
Sonu Verma, ${ }^{1}$ Tutul Biswas, ${ }^{2}$ and Tarun Kanti Ghosh ${ }^{1}$ \\ 1 Department of Physics, Indian Institute of Technology-Kanpur, Kanpur-208 016, India \\ 2 Department of Physics, University of North Bengal, Raja Rammohunpur-734 013, India
}

(Dated: July 12, 2019)

\begin{abstract}
Noncentrosymmetric metals such as $\mathrm{Li}_{2}\left(\mathrm{Pd}_{1-x} \mathrm{Pt}_{x}\right)_{3} \mathrm{~B}$ have different Fermi surface topology below and above the band touching point where spin-degeneracy is not lifted by the spin-orbit coupling. We investigate thermoelectric and optical response as probes for this Fermi surface topology change. We show that the chemical potential displays a dimensional crossover from a three-dimensional to one-dimensional characteristics as the descending Fermi energy crosses the band touching point. This dimensional crossover is due to the existence of different Fermi surface topology above and below the band touching point. We obtain an exact expression of relaxation time due to short-range scatterer by solving Boltzmann transport equations self-consistently. The thermoelctric power and figure of merit are significantly enhanced as the Fermi energy goes below the band touching point owing to the underlying one-dimensional-like nature of noncentrosymmteric bulk metals. The value of thermoelectric figure of merit goes beyond two as the Fermi energy approaches to the van Hove singularity for lower spin-orbit coupling. Similarly, the studies of the zero-frequency and finitefrequency optical conductivities in the zero-momentum limit reflect the nature of topological change of the Fermi surface. The Hall coefficient and optical absorption width exhibit distinct signatures in response to the changes in Fermi surface topology.
\end{abstract}

PACS numbers:

\section{INTRODUCTION}

Breaking of inversion symmetry has far reaching consequences in condensed matter physics. It gives rise to spin-orbit interaction (SOI), which itself serves as the backbone of the rich fast-growing field of spintronics ${ }^{1-3}$. At the interface of semiconductor heterostructures, the inversion symmetry is broken due to the band mismatch/external electric field, thus giving rise to a particular type of SOI known as Rashba spin-orbit interaction (RSOI $)^{4,5}$. Besides the breaking of inversion symmetry in bulk semiconductors having zinc blende structures causes Dresselhaus spin-orbit interaction ${ }^{6}$. The RSOI has potential applications in developing spintronic devices as its strength is externally tunable ${ }^{7}$ and therefore it is mostly studied. It is revealed that the RSOI can host a plethora of exotic phenomena such as dissipationless spin current ${ }^{8,9}$, spin Hall effect ${ }^{10-15}$, spin-orbit torque $^{15,16}$ and spin galvanic effect ${ }^{15,17}$.

Angle resolved photo emission spectroscopy has confirmed the existence of large spin splitting in several systems such as $\mathrm{Bi} / \mathrm{Ag}(111)$ surface alloy ${ }^{18}$, topological insulators like $\mathrm{Bi}_{2} \mathrm{Se}_{3}$ etc. ${ }^{19,20}$. The spin-orbit coupling strength found in these systems are larger in magnitude at least by two orders than that found in conventional semiconductor heterostructures. The recent discovery of giant $\mathrm{RSOI}^{21-25}$ in three-dimensional (3D) polar semiconductor $\mathrm{BiTeX}(\mathrm{X}=\mathrm{Cl}, \mathrm{Br}, \mathrm{I})$ has triggered immense investigations in the field of spintronics both theoretically and experimentally. The surface states of BiTeX exhibit large Rashba splitting as a result of surface-induced asymmetry. The origin of giant RSOI in the bulk of such materials has been unveiled by $\mathbf{k} \cdot \mathbf{p}$ perturbation analysis ${ }^{22}$ and is attributed to the distinct crystal structure of BiTeX and large $\mathrm{SOI}$ of $\mathrm{Bi}$. Itinerant electrons also experience strong RSOI in B20 compounds ${ }^{26}$ and noncentrosymmetric metals such as $\mathrm{Li}_{2}\left(\mathrm{Pd}_{1-x} \mathrm{Pt}_{x}\right)_{3} \mathrm{~B}^{27}$. The material $\mathrm{Li}_{2}\left(\mathrm{Pd}_{1-x} \mathrm{Pt}_{x}\right)_{3} \mathrm{~B}$ exhibits superconductivity ${ }^{28,29}$ as result of inversion symmetry breaking, whereas B20 compounds $^{30-33}$ host nontrivial spin textures including spin helix and magnetic skyrmions. The spinmomentum locking in $\mathrm{Li}_{2}\left(\mathrm{Pd}_{1-x} \mathrm{Pt}_{x}\right)_{3} \mathrm{~B}$ has distinct structure than that in the BiTeX family. Therefore, this noncentrosymmetric material has drawn immense interest from the perspective of superconductivity ${ }^{34}$, Kerr rotation $^{27,35}$, spin susceptibility, ${ }^{36}$ and Ruderman-KittelKasuya-Yosida interaction ${ }^{37}$.

To probe electronic states in BiTeX materials various investigations have been performed recently in the context of transport ${ }^{38-44}$, thermoelectric ${ }^{45,46}$, and optical $^{40,47-49}$ response. In addition there have also been theoretical and experimental studies ${ }^{50,51}$ where magnetic susceptibility of these systems changes its nature from paramagnetic to diamagnetic as Fermi energy crosses the band touching point from below. However, such studies are still missing in the case of spin-orbit coupled noncentrosymmetric metal like $\mathrm{Li}_{2}\left(\mathrm{Pd}_{1-x} \mathrm{Pt}_{x}\right)_{3} \mathrm{~B}$, which has different Fermi surface topology below and above the band touching point. This lack of information motivates us to address the issue that whether the thermoelectric and optical probes can be used to extract the information about the topology of the Fermi surface in noncentrosymmetric metals like $\mathrm{Li}_{2}\left(\mathrm{Pd}_{1-x} \mathrm{Pt}_{x}\right)_{3} \mathrm{~B}$.

Transport properties of spin-orbit coupled condensed matter systems at low temperature is of great interest for 
various reasons. It is of primary interest to reduce the thermal conductivity to obtain high thermoelectric figure of merit being inversely proportional to the thermal conductivity. The thermal energy is transported by the electrons as well as phonons. At temperatures much smaller than the Debye temperature $\theta_{D}$, number of phonons participating in thermal transport will be very small and electronic thermal conductivity will dominate over the lattice counterparts. For instance, in B20 compounds like $\mathrm{Fe}_{1-x} \mathrm{Co}_{x} \mathrm{Si}^{52} \theta_{D}$ is around $350 \mathrm{~K}$ and in $\mathrm{MnGe}, \mathrm{CoGe}^{53}$ $\theta_{D}$ is about (269-281) K. Therefore, in this work we consider the temperature around $(5-20) \mathrm{K}$ which is much smaller than $\theta_{D}$. Moreover, it is essential to have low temperature of the system so that thermal energy is always less than the spin splitting energy which is required to control spin of a charge carrier for spintronic device applications.

In this work, we provide a systematic study of thermoelectric transport coefficients and optical responses in noncentrosymmetric metals. We find that the chemical potential exhibits a dimensional crossover from a $3 \mathrm{D}$ - to 1D-like characteristics as the Fermi energy goes below the band touching point (BTP). This feature is attributed to the existence of different Fermi surface topology above and below the BTP. We obtain exact expression of relaxation time assuming short-range electron-impurity scattering, by solving the Boltzmann transport equations including interband scattering self-consistently. We provide results of all thermoelectric transport coefficients. The thermoelectric power and figure of merit are significantly enhanced below BTP owing to the underlying 1D-like nature of this system as a consequence of change in Fermi surface topology. We obtain a remarkable value, more than 2 of thermoelectric figure of merit for $\alpha=0.2 \mathrm{eV}$-nm at low density below BTP. Similarly, the studies of the zero-frequency and finite-frequency optical conductivities shed some light on the nature of spinsplit energy gap and would help to extract the spin-orbit coupling strength. We find that Hall coefficient and optical absorption width respond differently to the change in the Fermi surface topology.

This paper is organized as follows. In Sec. II, we provide a discussion on the ground state properties of the physical system considered. In Sec. III, we discuss various thermoelectric properties. Sec. IV includes information of the Drude weight, Hall coefficient, and finitefrequency optical conductivity. We summarize our main results in Sec. V.

\section{GROUND STATE PROPERTIES}

We consider conduction electrons in a 3D noncentrosymmetric metal. As mentioned in the introduction, the usual examples of noncentrosymmetric metals are $\mathrm{Li}_{2}\left(\mathrm{Pd}_{3-x} \mathrm{Pt}_{x}\right) \mathrm{B}$ and $\mathrm{B} 20$ compounds. All these materials possess cubic crystal structure. In this particular lattice geometry, the low-energy conduction electrons can be effectively described by the following Hamiltonian based on symmetry analysis ${ }^{26,34}$ :

$$
H=\frac{\hbar^{2} \mathbf{k}^{2}}{2 m^{*}} \sigma_{0}+\alpha \boldsymbol{\sigma} \cdot \mathbf{k},
$$

where $m^{*}$ is the effective mass of electron, $\sigma_{0}$ is $2 \times 2$ identity matrix, $\boldsymbol{\sigma}=\left\{\sigma_{x}, \sigma_{y}, \sigma_{z}\right\}$ is Pauli spin matrix, $\mathbf{k}=\left\{k_{x}, k_{y}, k_{z}\right\}$ with $k=\sqrt{k_{x}^{2}+k_{y}^{2}+k_{z}^{2}}$ is the electron's wavevector, and $\alpha$ is the strength of the RSOI. The RSOI term in Eq. (1) has the form $\boldsymbol{\sigma} \cdot \mathbf{k}$ in which $k_{x}, k_{y}$, and $k_{z}$ are intertwined with $\sigma_{x}, \sigma_{y}$, and $\sigma_{z}$, respectively. This distinct spin-momentum locking is absent in 2D Rashba systems and 3D Rashba semiconductors such as BiTeX and therefore gives rise to particular Fermi surface topology, different than other Rashba systems, which will be discussed shortly. Note that the Hamiltonian commutes with the helicity operator $\mathbf{k} \cdot \boldsymbol{\sigma} / k$ so that its eigenvalues \pm 1 are good quantum numbers. Thus, the eigenstates of the above Hamiltonian can be obtained as eigenstates of the helicity operator modulated by a plane wave like $\psi_{\mathbf{k}}^{\lambda}(\mathbf{r})=\phi_{\mathbf{k}}^{\lambda} e^{i \mathbf{k} \cdot \mathbf{r}} / \sqrt{V}$, where $V$ is volume of the system, $\lambda= \pm$ represents two opposite helicity, and $\phi_{\mathbf{k}}^{\lambda}$ is helicity eigenstate which takes the following forms: $\phi_{\mathbf{k}}^{+}=\left[\begin{array}{ll}\cos (\theta / 2) & e^{i \phi} \sin (\theta / 2)\end{array}\right]^{\mathcal{T}}$ for $\lambda=+$ and $\phi_{\mathbf{k}}^{-}=\left[\begin{array}{ll}\sin (\theta / 2) & -e^{i \phi} \cos (\theta / 2)\end{array}\right]^{\mathcal{T}}$ for $\lambda=-$, with $\mathcal{T}$ being the transpose operation. Here, $\theta$ and $\phi$ are the polar and azimuthal angle, respectively, which represent the orientation of $\mathbf{k}$. The energy dispersion consists of two spin-split bands corresponding to $\lambda= \pm$ having the structure $E_{\mathbf{k} \lambda}=\hbar^{2} k^{2} /\left(2 m^{*}\right)+\lambda \alpha k$. This dispersion is depicted in Fig. 1(a). The full bandstructure calculations in $\mathrm{Li}_{2} \mathrm{Pd}_{3} \mathrm{~B}^{54}$ shows that the low-energy bands around the $\Gamma$ point with spin-orbit coupling are similar to Fig. 1(a). Comparing with the band structure calculations, the model Hamiltonian appears to be valid for $-30 \mathrm{meV}<E<0.1 \mathrm{eV}$ with a typical value of $\alpha=0.1$ $\mathrm{eV} \mathrm{nm.}$

The band $E_{\mathbf{k}-}$ has a nonmonotonous behavior for $E<0$ and attains a minimum value $E_{\min }=-E_{\alpha}=$ $-\hbar^{2} k_{\alpha}^{2} /\left(2 m^{*}\right)$ at $k=k_{\alpha}=m^{*} \alpha / \hbar^{2}$. In Figs. 1(b) and $1(\mathrm{c})$, constant energy surfaces are shown for $E>0$ and $E<0$, respectively. The wavevectors corresponding to $E>0$ are given by $k_{\lambda}=-\lambda k_{\alpha}+\sqrt{k_{\alpha}^{2}+2 m^{*} E / \hbar^{2}}$. Here, $k_{ \pm}$represent the radii of the two concentric spherical constant energy surfaces as shown in Fig. 1(b). For $E>0$, the topology of the Fermi surface has convexconvex shape on $\lambda=+$ and $\lambda=-$ bands, respectively. The corresponding density of states for $\lambda= \pm$ bands are given as

$$
D_{\lambda}^{>}(E)=\frac{1}{4 \pi^{2}}\left(\frac{2 m^{*}}{\hbar^{2}}\right)^{\frac{3}{2}}\left[\frac{E+2 E_{\alpha}}{\sqrt{E+E_{\alpha}}}-\lambda \sqrt{4 E_{\alpha}}\right] .
$$

On contrary for $E<0$, there exists only one energy band $E_{\mathbf{k}}^{-}$and the topology of energy surface changes completely as compared to $E>0$. For $E<0$, the topology of the Fermi surface has concave-convex shape on 
the inner and outer branches, respectively. The crosssection of the Fermi surface for $E<0$ is shown in Fig. 1 (c). This is characterized by the following wave vectors $k_{\eta}=k_{\alpha}-(-1)^{\eta-1} \sqrt{k_{\alpha}^{2}+2 m^{*} E / \hbar^{2}}, \eta=1,2$. It is worthy to mention that $0 \leq k_{1} \leq k_{\alpha}$ and $k_{\alpha} \leq k_{2} \leq 2 k_{\alpha}$. The region between two concentric spherical shells with the inner radius $k_{1}$ and outer radius $k_{2}$ contains the following available density of states

$D_{\eta}^{<}(E)=\frac{1}{4 \pi^{2}}\left(\frac{2 m^{*}}{\hbar^{2}}\right)^{\frac{3}{2}}\left[\frac{E+2 E_{\alpha}}{\sqrt{E+E_{\alpha}}}-(-1)^{\eta-1} \sqrt{4 E_{\alpha}}\right]$.

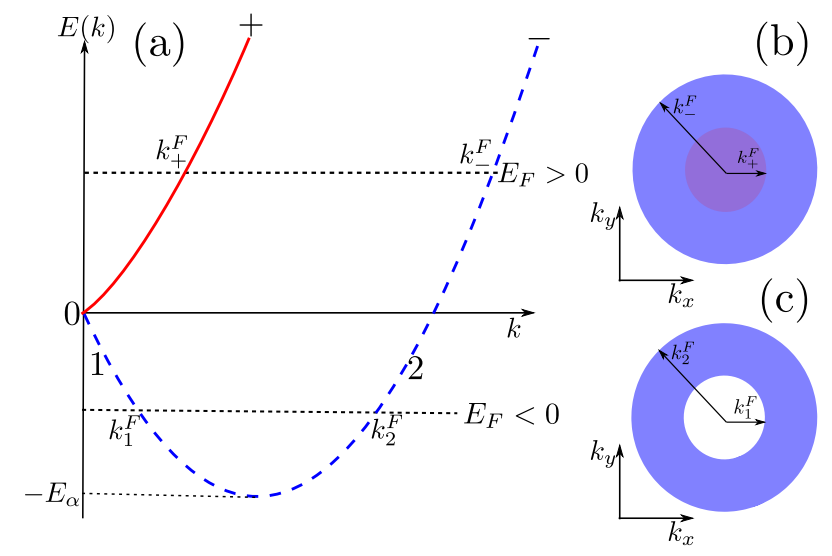

FIG. 1: (a) Spin-split energy bands of the noncentrosymmetric metals: The two bands touch at $k=0$ which is known as band touching point (BTP). (b) Cross-sections of the Fermi surfaces for $E_{F}>0$ and $E_{F}<0$. The Fermi surface topology has convex-convex shape and concave-convex shape for $E_{F}>0$ and $E_{F}<0$, respectively. The Fermi surface topology changes at band touching point (at $E_{F}=0$ ).

It is interesting to note that there is an inverse squareroot divergence of $D_{\eta}^{<}(E)$ as $E \rightarrow-E_{\alpha}$, similar to the van Hove singularity in conventional one-dimensional systems as well as in 2D Rashba systems. The large DOS in the very low-density $\left(E \rightarrow-E_{\alpha}\right)$ limit is due to the nonvanishing spherical energy surfaces with the radii $k_{1,2}$ approach to $k_{\alpha}$, and vanishing velocity as $\mathbf{v}(\mathbf{k})=(1 / \hbar) \nabla_{\mathbf{k}} E_{\mathbf{k}-} \propto \sqrt{E+E_{\alpha}}$.

For a given electron density $n_{e}$, the chemical potential $\mu$ at temperature $T$ can be obtained from the normalization condition,

$n_{e}=\sum_{\eta} \int_{-E_{\alpha}}^{0} f\left(E_{\eta}\right) D_{\eta}^{<}(E) d E+\sum_{\lambda} \int_{0}^{\infty} f\left(E_{\lambda}\right) D_{\lambda}^{>}(E) d E,(4)$

where $f(E)=\left[e^{(E-\mu) /\left(k_{B} T\right)}+1\right]^{-1}$ is the Fermi-Dirac distribution function. In the $T \rightarrow 0$ limit, we obtain Eq. $(4)$ in the following form to extract Fermi energy $E_{F}$

$$
3 \pi^{2} n_{e}=\sqrt{k_{\alpha}^{2}+\frac{2 m^{*} E_{F}}{\hbar^{2}}}\left[4 k_{\alpha}^{2}+\frac{2 m^{*} E_{F}}{\hbar^{2}}\right] .
$$

Note that with $\alpha=0$ Eq. (5) correctly reproduces the known result of the Fermi energy for conventional 3DEG:
$E_{F}^{0}=\frac{\hbar^{2}}{2 m^{*}}\left(3 \pi^{2} n_{e}\right)^{2 / 3}$. The topology of the Fermi surface changes at $n_{e}=n_{t}$ with $n_{t}=4 k_{\alpha}^{3} / 3 \pi^{2}$. For a given $\alpha$, $E_{F}<0$ when $n_{e}<n_{t}$ and $E_{F}>0$ when $n_{e}>n_{t}$. At finite temperature, we obtain the chemical potential $\mu$ by solving Eq. (4) numerically for $\alpha=0.1 \mathrm{eV}-\mathrm{nm}$. For three different temperatures, namely, $T=5,10$, and $20 \mathrm{~K}$, the difference between the chemical potential and Fermi energy is shown in Fig. 2. We find that $\mu-E_{F}$ exhibits a dimensional crossover as the Fermi energy changes its sign. For instance, $\mu-E_{F}$ is negative when $E_{F}>0$. This feature corresponds to the nature of chemical potential in $3 \mathrm{D}$ case. On the contrary, for $E_{F}<0, \mu-E_{F}$ is positive. This behavior is clearly a hallmark of $\mu$ in $1 \mathrm{D}$ case. However, this indirect signature of dimensional crossover is not clearly seen from the structures of the density of states corresponding to $E_{F}>0$ and $E_{F}<0$.

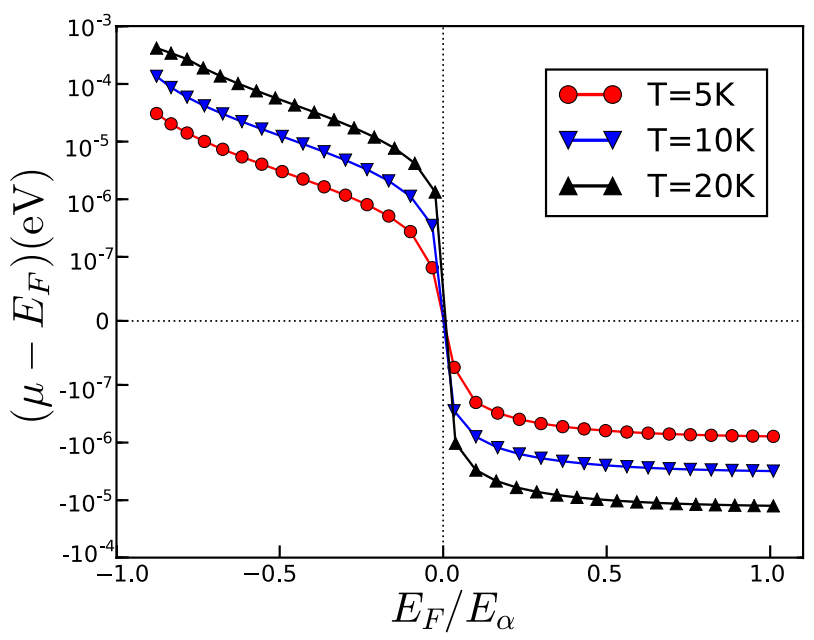

FIG. 2: Difference between $\mu$ and $E_{F}$ as a function of $E_{F}$ at a fixed $\alpha=10^{-10} \mathrm{eV} \mathrm{m}$ for $T=5,10$, and $20 \mathrm{~K}$. The change in sign of $\mu-E_{F}$ at $E_{F}=0$ implies the dimensional crossover.

\section{THERMOELECTRIC TRANSPORT}

\section{A. General formalism}

We consider the physical system is subjected to a spatially uniform electric field $\boldsymbol{\Xi}$ and a temperature gradient $\boldsymbol{\nabla} T$. The magnitude of the external electric field and temperature gradient are chosen in such a way that the linear response theory holds.

The electronic and thermal current densities are given by $\mathbf{j}=-e \sum_{\xi} \mathbf{v}_{\xi} f_{\xi}$ and $\mathbf{j}_{\text {th }}=\sum_{\xi}\left(E_{\xi}-\mu\right) \mathbf{v}_{\xi} f_{\xi}$, respectively. Here, $\xi$ defines the set of all quantum numbers, i.e., $\xi \equiv(\lambda, \eta, \mathbf{k})$, and $\mathbf{v}_{\xi}$ is the group velocity of the electron in the quantum state $\xi$. Within the context of linear response theory, the nonequilibrium distribution 
function $f_{\xi}$ is given by

$$
f_{\xi}=f_{\xi}^{0}+\tau_{\xi}\left(-\frac{\partial f_{\xi}^{0}}{\partial E_{\xi}}\right) \mathbf{v}_{\xi} \cdot\left[-e \Xi^{*}+\frac{E_{\xi}-\mu}{k_{B} T}(-\nabla T)\right]
$$

where $f_{\xi}^{0}=\left[e^{\left(E_{\xi}-\mu\right) /\left(k_{B} T\right)}+1\right]^{-1}$ is the Fermi-Dirac distribution function at equilibrium, $\tau_{\xi} \equiv \tau\left(E_{\xi}\right)$ is the energy dependent relaxation time, and $\boldsymbol{\Xi}^{*}=\boldsymbol{\Xi}+\nabla \mu / e$ is the effective electric field.

The current densities can be found together in the following form ${ }^{55}$ :

$$
\left(\begin{array}{c}
\mathbf{j} \\
\mathbf{j}_{\mathrm{th}}
\end{array}\right)=\left(\begin{array}{ll}
L_{11} & L_{12} \\
L_{21} & L_{22}
\end{array}\right)\left(\begin{array}{c}
\boldsymbol{\Xi}^{*} \\
-\boldsymbol{\nabla} T
\end{array}\right)
$$

where $L_{p q}$ 's are defined as $L_{11}=\mathcal{L}^{(0)}, L_{21}=T L_{12}=$ $-\mathcal{L}^{(1)} / e$, and $L_{22}=\mathcal{L}^{(2)} /\left(e^{2} T\right)$ with

$$
\mathcal{L}_{\nu \nu^{\prime}}^{(r)}=e^{2} \sum_{\xi} \tau_{\xi} v_{\nu}(\xi) v_{\nu^{\prime}}(\xi)\left(E_{\xi}-\mu\right)^{r}\left(-\frac{\partial f_{\xi}^{0}}{\partial E_{\xi}}\right),
$$

with $\nu, \nu^{\prime}=x, y, z$. Note that different combinations of $L_{p q}$ can be used to define the thermoelectric coefficients like electrical conductivity, thermopower, and thermal conductivity. For instance, $L_{11}$ can be identified as the electrical conductivity, whereas the thermopower and the thermal conductivity are defined as $S=L_{21} / L_{11}$ and $\kappa=L_{22}-L_{21}\left(L_{11}\right)^{-1} L_{12}$, respectively. We are going to determine $\mathcal{L}_{\nu \nu^{\prime}}^{(r)}$ in a more explicit way, relevant for the present scenario. Let us now restrict ourselves to consider that the scattering mechanisms, responsible for thermoelectric transport, are due to the presence of weak spinindependent disorders distributed throughout the sample with an average density $n_{\mathrm{imp}}$. The short-range disorder potential is given by $U(\mathbf{r})=U_{0} \sum_{i} \delta\left(\mathbf{r}-\mathbf{r}_{\mathbf{i}}\right)$, where $U_{0}$ is the strength of the potential having dimension of energy times volume and $\mathbf{r}_{i}$ is the position of the $i$-th scatterer. Note that the present situation is an example of isotropic case since the energy spectrum depends only on the magnitude of $\mathbf{k}$. In this case, $\mathcal{L}_{\nu \nu^{\prime}}^{(r)}=\mathcal{L}_{\nu \nu}^{(r)} \delta_{\nu \nu^{\prime}} \equiv \mathcal{L}^{(r)}$. Also the expectation value of $\hat{v}_{\nu}(\mathbf{k})$ with respect to the state $\lambda$ is $\left\langle\hat{v}_{\nu}(\mathbf{k})\right\rangle_{\lambda}=\frac{\hbar k_{\nu}}{m^{*}}+\lambda \frac{\alpha}{\hbar} \frac{k_{\nu}}{k}$. Equation (8) can be rewritten further as

$$
\begin{aligned}
\mathcal{L}^{(r)} & =\frac{e^{2}}{(2 \pi)^{3}} \sum_{\lambda= \pm} \int_{\mu>0} d^{3} k \tau_{\lambda}\left(E_{\mathbf{k} \lambda}\right)\left\langle\hat{v}_{\nu}(\mathbf{k})\right\rangle_{\lambda}^{2}\left(E_{\mathbf{k} \lambda}-\mu\right)^{r}\left(-\frac{\partial f_{\mathbf{k} \lambda}^{0}}{\partial E_{\mathbf{k} \lambda}}\right) \\
& +\frac{e^{2}}{(2 \pi)^{3}} \sum_{\eta=1,2} \int_{\mu<0} d^{3} k \tau_{\eta}\left(E_{\mathbf{k}-}\right)\left\langle\hat{v}_{\nu}(\mathbf{k})\right\rangle_{-}^{2}\left(E_{\mathbf{k}-}-\mu\right)^{r}\left(-\frac{\partial f_{\mathbf{k}-}^{0}}{\partial E_{\mathbf{k}-}}\right) .
\end{aligned}
$$

The relaxation time $\tau_{\lambda(\eta)}$ in Eq. (9) can be determined using the framework of semiclassical Boltzmann transport theory including interband scattering for multiband system. When Fermi energy lies below the BTP, an unusual intraband scattering (interbranch and intrabranch scatterings) arises due to the concave-convex shaped Fermi surface topology as shown in Fig. 1(c). For the present case, we solve the Boltzmann transport equation including the interband/interbranch scattering term self-consistently (see the Appendix for detail derivation) to find

$$
\tau_{\lambda}(E)=\frac{\hbar}{\pi n_{\mathrm{imp}} U_{0}^{2}}\left[\frac{D_{\lambda}^{>}(E)}{\left[D_{T}^{>}(E)\right]^{2}}+\frac{1}{2 D_{T}^{>}(E)}\right]
$$

for $E>0$ and

$$
\tau_{\eta}(E)=\frac{\hbar}{\pi n_{\mathrm{imp}} U_{0}^{2}}\left[\frac{D_{\eta}^{<}(E)}{\left[D_{T}^{<}(E)\right]^{2}}+\frac{1}{2 D_{T}^{<}(E)}\right]
$$

for $E<0$. Here, $D_{T}^{>}(E)=D_{+}^{>}(E)+D_{-}^{>}(E)$ and $D_{T}^{<}(E)=D_{1}^{<}(E)+D_{2}^{<}(E)$ are the total density of states for $E>0$ and $-E_{\alpha}<E<0$, respectively. Note that the expressions of $\tau(E)$ for $E>0$ and $E<0$ share the same mathematical structures. This help us to find $\mathcal{L}^{(r)}$ in a more compact form,

$$
\mathcal{L}^{(r)}=\frac{4}{3} \frac{e^{2}}{h l_{0}}\left(k_{B} T\right)^{r} X_{r}
$$

where $l_{0}=m^{*} n_{\text {imp }} U_{0}^{2} /\left(\hbar^{2} k_{B} T\right), X_{r}=\int_{-x_{\alpha}}^{\infty} \mathcal{L}(x)(x-$ $\tilde{\mu})^{r} d x$, with $x=E /\left(k_{B} T\right)$ and $\tilde{\mu}=\mu /\left(k_{B} T\right)$. Here $\mathcal{L}(x)$ is given by

$$
\mathcal{L}(x)=f_{x}\left(1-f_{x}\right)\left(x+x_{\alpha}\right)\left[1+2 \frac{x_{\alpha}\left(x+x_{\alpha}\right)}{\left(x+2 x_{\alpha}\right)^{2}}\right],
$$

with $f_{x}=\left[e^{x-\tilde{\mu}}+1\right]^{-1}$ and $x_{\alpha}=E_{\alpha} /\left(k_{B} T\right)$.

\section{B. Results}

Here we discuss the behavior of different thermoelectric coefficients obtained via the numerical solution of Eq. 
(12). For numerical calculation we consider following material parameters : effective mass of electron $m^{*}=0.5 m_{e}$, $m_{e}$ is the free electron mass and $\alpha_{0}=10^{-10} \mathrm{eVm}$.

Let us begin with the behavior of the electrical conductivity $\sigma=L_{11}=\mathcal{L}^{(0)}$. From Eq. (12), we explicitly have $\sigma=4 e^{2} X_{0} /\left(3 h l_{0}\right)$. Figure 3(a) depicts the variation of $\sigma$ with chemical potential for $\alpha=0.2 \alpha_{0}, 0.5 \alpha_{0}$, and $\alpha_{0}$ at $T=5 \mathrm{~K}$. The conductivity increases monotonically with $\mu$. For higher values of $\alpha$, the enhancement of $\sigma$ is significant. In the $T \rightarrow 0$ limit, we obtain the following analytical expression of $\sigma$ as (see the Appendix for detail derivation)

$$
\sigma=\frac{e^{2}}{h} \frac{2 \hbar^{2}\left(E_{\alpha}+E_{F}\right)}{m^{*} n_{\mathrm{imp}} U_{0}^{2}}\left[1-\frac{E_{F}^{2}}{3\left(2 E_{\alpha}+E_{F}\right)^{2}}\right]
$$

which shows that the zero temperature conductivity increases linearly with the Fermi energy. Note that the characteristics of $\sigma$ with $E_{F}$ in the $E_{F}>0$ and $E_{F}<0$ are the same. This is different from the behavior of $\sigma$ with $E_{F}$ in 2 D Rashba systems ${ }^{56}$ in which it was found that the zero temperature conductivity depends on the Fermi energy in linear (quadratic) fashion for $E_{F}>0$ $\left(E_{F}<0\right)$. This feature was attributed to the fact that $\sigma(E)$ cannot be continuously differentiated at $E_{F}=0$. In that 2D Rashba system density of states of individual bands/branches depends linearly on wave vector, which immediately implies that total density of states below and above BTP have different energy dependence. So relaxation time having similar structures below and above the BTP will have different energy dependence. As a result of this the electrical conductivity being proportional to the relaxation time shows different energy dependence below and above BTP. However, in the present context, Eq. (14) clearly demonstrates that $\sigma(E)$ is a continuously differentiable function at $E_{F}=0$. This fact helped us to find same analytical structures of $\sigma\left(E_{F}\right)$ in both $E_{F}>0$ and $E_{F}<0$ regions. From Eq. (14) it is evident that $\sigma \propto E_{\alpha}$ at $E_{F}=0$. The signature of this feature is also reflected in Fig. 3(a). The reason of not seeing the direct indication of change in Fermi surface topology in electrical conductivity in our case is that the density of states depends on square of the wave vector as a consequence of the $3 \mathrm{D}$ system. It implies that the form of total density of states, and hence relaxation time will have the same energy dependence below and above the BTP (see the Appendix for more details).

The thermal conductivity $\kappa$ is obtained as $\kappa=$ $\left(4 / \pi^{2}\right) \kappa_{0} \kappa_{I}$, where $\kappa_{0}=e^{2} L_{0} T /\left(h l_{0}\right)$, with $L_{0}=$ $\left(\pi^{2} / 3\right)\left(k_{B} / e\right)^{2}$ as the Lorentz number for 3D electron gas and $\kappa_{I}=X_{2}-X_{1}^{2} / X_{0}$. The variation of the thermal conductivity with the chemical potential is depicted in Fig. 3(b). The thermal conductivity behaves with the chemical potential in a similar fashion as the electrical conductivity.

We obtain the thermopower through explicit calculation as $S=-\left(k_{B} / e\right) S_{I}$, where $S_{I}=X_{1} / X_{0}$. In Fig. 4 , we show the dependence of $S$ on $\mu$ for $\alpha=0.2 \alpha_{0}, 0.5 \alpha_{0}$, and $\alpha_{0}$. The thermopower is large at lower values of $\alpha$
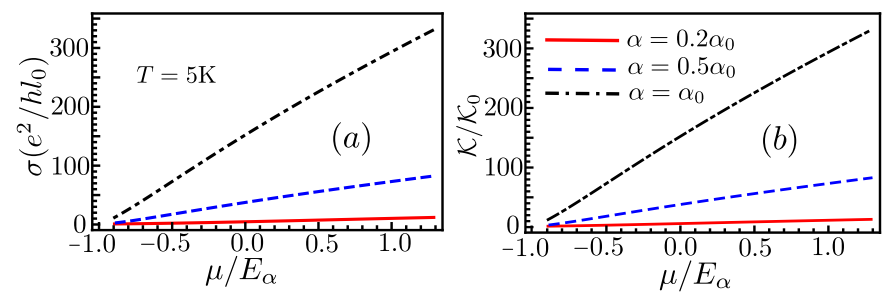

FIG. 3: Plots of (a) electrical conductivity $\sigma$ and (b) thermal conductivity $\kappa$ as a function of chemical potential $\mu$ at temperature $T=5 \mathrm{~K}$ for three different values of $\alpha$.

as compared to higher $\alpha$. In the region below $E_{F}=0$ magnitude of thermopower changes rapidly with $\mu$. For $E_{F}>0$ the rate of change of $S$ with $\mu$ is slow compared to the previous case. Moreover, for higher $\alpha$, the thermopower attains a saturation value when $\mu>0.5 E_{\alpha}$. In the $\left|E_{F}\right| \gg k_{B} T$ limit, using the Mott relation ${ }^{55}$

$$
S_{\mathrm{Mott}}=-\left.\frac{\pi^{2} k_{B}^{2} T}{3 e} \frac{d \ln \sigma(E)}{d E}\right|_{E=E_{F}},
$$

one may obtain the following expression of thermoelectric power:

$$
S_{\mathrm{Mott}}=-\frac{\pi^{2} k_{B} T}{3 e\left(E_{F}+E_{\alpha}\right)}\left[1-\frac{2 E_{F} E_{\alpha}\left(E_{F}+E_{\alpha}\right)}{\left(E_{F}+2 E_{\alpha}\right) \xi_{F}}\right],
$$

where $\xi_{F}=E_{F}^{2}+6 E_{\alpha} E_{F}+6 E_{\alpha}^{2}$. The inset of Fig. 4 shows the variation of $S / S_{\text {Mott }}$ as a function of chemical potential for different values of $\alpha$ at $T=5 \mathrm{~K}$. For lower values of $\alpha$, namely, $\alpha=0.2 \alpha_{0}$, the Mott relation is valid only when $\mu>0.5 E_{\alpha}$. The degree of validity of the Mott relation increases with $\alpha$. For $\alpha=\alpha_{0}$, the Mott formula is satisfied almost in the entire range of the chemical potential considered. It is known ${ }^{55,56}$ that the validity of Mott relation or the Sommerfeld expansion depends on the following simultaneous conditions: (1) $T \ll T_{F}$ where $T_{F}=E_{F} / k_{B}$ is the Fermi temperature and (2) whether the Taylor expansion of $\sigma(E)$ about $E=\mu$ is possible or not. In our case, Condition (2) is satisfied always because $\sigma(E)$ is a continuously differentiable function. Therefore, we attribute the breakdown of Mott relation (as shown in Fig. 4) to the breakdown of the validity of Condition (1).

The thermoelectric figure of merit is a dimensionless number which measures the efficiency of the thermoelectric performance of a material. It is defined as $Z T=S^{2} \sigma T / \kappa$. In general, the symbol $\kappa$ used in the definition of $Z T$ stands for the total thermal conductivity which is a sum of electronic and phononic contribution. But, in the temperature regime we are focusing on now, the electronic thermal conductivity dominates over the thermal counterparts. Therefore, we neglect lattice contribution to the thermal conductivity and proceed with the electronic counterpart for the subsequent calculation of $Z T$. A good thermoelectric material needs to possess 


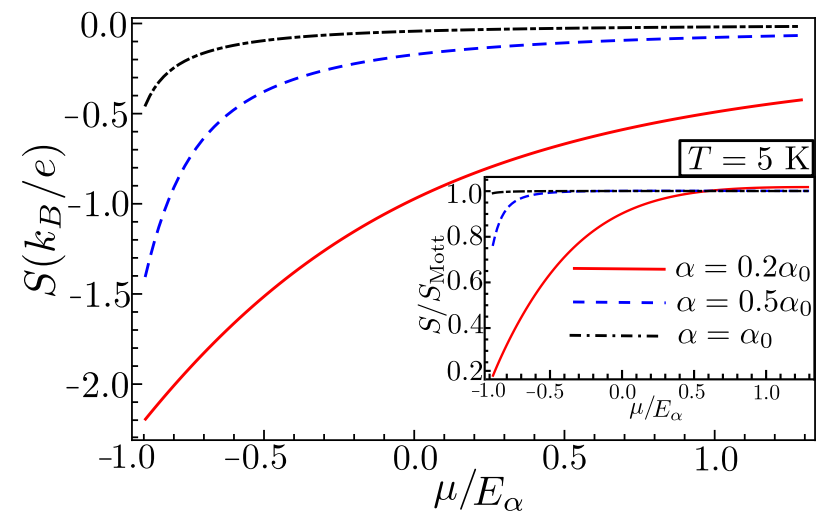

FIG. 4: Plots of the thermopower $S$ as a function of chemical potential $\mu$ at temperature $T=5 \mathrm{~K}$ for three different values of $\alpha$.

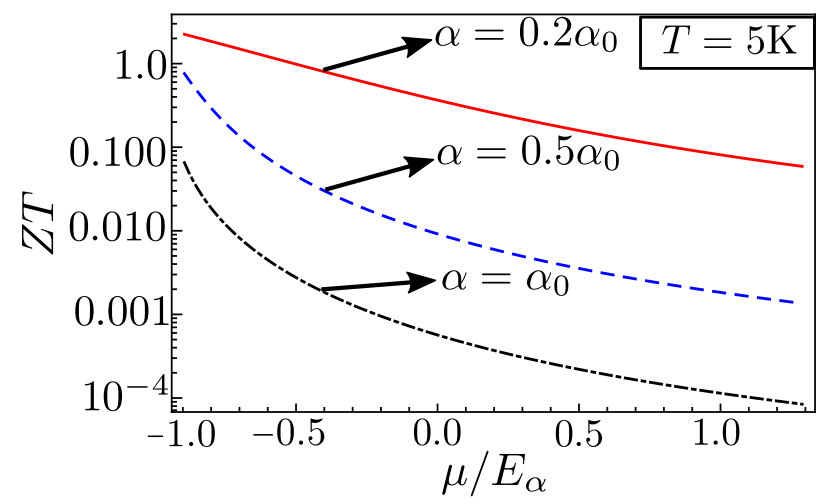

FIG. 5: Plots of the thermoelectric figure of merit $Z T$ as a function of chemical potential $\mu$ at temperature $T=5 \mathrm{~K}$ for three different values of $\alpha$.

following properties : large electrical conductivity, high thermopower, and low thermal conductivity. For the current scenario, we obtain $Z T$ explicitly as $Z T=S_{I}^{2} X_{0} / \kappa_{I}$. In Fig. 5 we show the dependence of $Z T$ on the chemical potential at $T=5 \mathrm{~K}$ for three different values of $\alpha$, namely, $\alpha=0.2 \alpha_{0}, 0.5 \alpha_{0}$, and $\alpha_{0}$. It is clear that $Z T$ behaves as a monotonically decreasing function of $\mu$ for each $\alpha$. The value of $Z T$ is higher at lower $\alpha$. This is obvious because the order of magnitude of $\sigma$ and $\kappa$ are almost same for each $\alpha$ whereas the thermopower is large at lower $\alpha$. In other words, the thermopower alone determines the behavior of $Z T$.

Note that for $\alpha=0.2 \alpha_{0}, Z T$ attains a remarkable value greater than 2 when the chemical potential lies far below the BTP. To explain this feature explicitly, in Fig. 6 we plot $D(E), D(E)\left(-\partial f^{0} / \partial E\right)$, and the integrand of $\mathcal{L}^{(1)}$ (which is proportional to $S$ ) as a function of energy for a particular $\alpha$, namely, $\alpha=\alpha_{0}$. We consider two different densities, namely, $n_{e}^{(1)}$ and $n_{e}^{(2)}$ which in turn correspond to chemical potential $\mu_{1}$ and $\mu_{2}$, respectively, at some constant temperature $T$. The chemical potential $\mu_{1}$ lies above the band touching point whereas $\mu_{2}$ falls well below of it. As expected the function $D(E)\left(-\partial f^{0} / \partial E\right)$ exhibit a peak whenever the energy matches with the chemical potential. For $n_{e}=n_{e}^{(1)}$, as expected the integrand of $\mathcal{L}^{(1)}$ changes sign when the energy crosses $\mu_{1}$ and exhibits a structure as shown by the shaded portion. It is hard to differentiate between the areas under the curves (shaded region in Fig. 6) below and above $\mu_{1}$. Therefore, when summed up it gives rise to negligible contribution to the thermoelectric power which is reflected in Fig. 4.

When $n_{e}=n_{e}^{(2)}$, the asymmetry between the magnitudes of $\mathcal{L}^{(1)}$ below and above $\mu_{2}$ can be visible. This increment in the asymmetry is responsible for the enhancement of thermopower below BTP. The amount of asymmetry increases as the chemical potential approaches $-E_{\alpha}$. As we tune the chemical potential from positive to negative value the amount of asymmetry increases due to the dimensional crossover from $3 \mathrm{D}$ to $1 \mathrm{D}$, which leads to an increase of thermopower as well as thermoelectric figure of merit. There have been several studies before $^{57,58}$, where it has been shown that the low dimensional systems lead to larger asymmetry in the density of states about the chemical potential, which give rise to larger thermopower and thermoelectric figure of merit and hence can be used for good thermoelectric device applications. Our result is consistent with these observations as we obtain larger value of figure of merit below the BTP due to the underlying 1D-like characteristics of our bulk 3D system. It can be verified that the amount of asymmetry will become more prominent for the cases of lower $\alpha$. This asymmetry explains the higher values of ZT obtained for low $\alpha$. Therefore, one has to struggle to fix both $\alpha$ and $n_{e}$ at reasonable values to use 3D noncentrosymmetric metal for good thermoelectric devices. Also the thermopower $S$ and figure of merit $Z T$ tend to diverge as $\mu$ approaches $-E_{\alpha}$. This fact is attributed to the presence of the van Hove singularity in the band structure, similar to quasi-1D systems ${ }^{58}$.

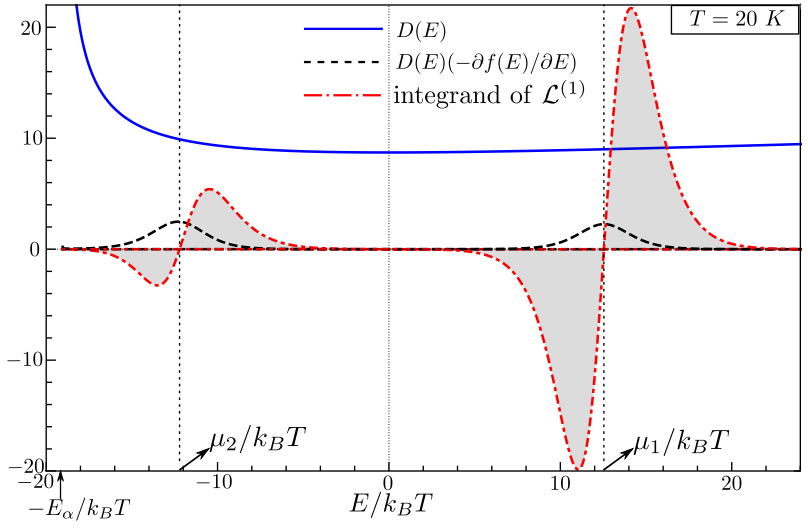

FIG. 6: Plots of $D(E), D(E)\left(-\partial f^{0} / \partial E\right)$, and the integrand of $\mathcal{L}^{(1)}$ as a function of $E$ for $\alpha=\alpha_{0}$. For a better visualization we consider $T=20 \mathrm{~K}$. 
We emphasize here that at very low density (as $E_{F} \rightarrow$ $-E_{\alpha}$ ), the singularity in the density of states may be broadened by disorder due to finite-band effects ${ }^{59}$. Such quantum effects must be considered using the Kubo formula $^{60}$, rather than semiclassical Boltzmann transport theory.

\section{ZERO-MOMENTUM OPTICAL CONDUCTIVITY}

In this section, we present optical signature of a change in the Fermi surface topology in noncentrosymmetric metals at $T=0$. The zero-frequency and finitefrequency optical conductivities are the manifestations of the intra-band and interband optical transitions, respectively. Consider the noncentrosymmetric metal is irradiated by a weak and spatially homogeneous electric field $\mathbf{E}=E_{0} e^{i \omega t} \hat{\boldsymbol{\nu}}$ oscillating with the frequency $\omega$ and amplitude $E_{0}$. The absorptive part of the charge optical conductivity tensor is given by $\operatorname{Re} \Sigma_{\nu \nu^{\prime}}(\omega)=$ $\delta(\omega) D_{w}^{\nu \nu^{\prime}}+\operatorname{Re} \sigma_{\nu \nu^{\prime}}(\omega)$, where $\nu, \nu^{\prime}=x, y, z$ and $D_{w}^{\nu \nu^{\prime}}$ is the Drude weight (charge stiffness). The semiclassical expression of the Drude weight ${ }^{55}$ is given by

$$
D_{w}^{\nu \nu^{\prime}}=\pi e^{2} \sum_{\lambda} \int \frac{d^{3} k}{(2 \pi)^{3}}\left\langle\hat{v}_{\nu}\right\rangle_{\lambda}\left\langle\hat{v}_{\nu^{\prime}}\right\rangle_{\lambda} \delta\left(E_{\lambda}(\mathbf{k})-E_{F}\right)
$$

This semiclassical expression has been successfully used in 2DEG with linear spin-orbit interaction as well as in $2 \mathrm{D}$ hole gas with $k$-cubic spin-orbit interaction ${ }^{61}$. The exact analytical expression of the Drude weight is obtained as

$$
D_{w}^{\nu \nu^{\prime}}=\frac{\pi e^{2} n_{e}}{m^{*}}\left[\frac{E_{F}+2 E_{\alpha}}{E_{F}+4 E_{\alpha}}\right] \delta_{\nu \nu^{\prime}} \theta\left(E_{F}+E_{\alpha}\right) .
$$

The longitudinal Drude weight is isotropic, i.e., $D_{w}^{x x}=$ $D_{w}^{y y}=D_{w}^{z z}=D_{w}$ and the off-diagonal Drude weight vanishes exactly. Setting $\alpha=0$ in the above equation, we get the standard result of the Drude weight for 3DEG, i.e., $D_{w}^{0}=\pi n_{e} e^{2} / m^{*}$. It shows that the spin-orbit coupling reduces the Drude weight as compared to the conventional 3DEG without spin-orbit coupling and further an increase in $\alpha$ decreases it more as shown in Fig. 7(a). We also see in Fig. 7(a) that similar to Drude conductivity, Drude weight also shows no signature of change in Fermi surface topology.

It has been shown ${ }^{62,63}$ that the Hall coefficient $R_{H}$ can be obtained from the Drude weight using the general expression

$$
R_{H}=-\frac{1}{e D_{w}} \frac{\partial D_{w}}{\partial n_{e}}
$$

This expression has been successfully used to calculate the Hall coefficient in various systems ${ }^{62-64}$. For the present system, the exact Hall coefficient is given by

$$
R_{H}=R_{H}^{0}\left[1+\frac{2 E_{\alpha}\left(E_{\alpha}+E_{F}\right)}{3\left(2 E_{\alpha}+E_{F}\right)^{2}}\right] \theta\left(E_{F}+E_{\alpha}\right),
$$

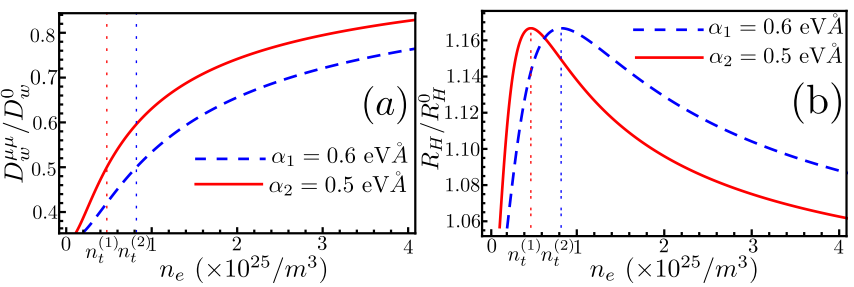

FIG. 7: Plots of the Drude weight $D_{w}^{\nu \nu^{\prime}}$ (a) and the Hall coefficient $R_{H}$ (b) as a function of carrier density $n_{e}$ for two different values of $\alpha$. Here $n_{t}^{(i=1,2)}=4 k_{\alpha_{i}} /\left(3 \pi^{2}\right)$ (with $i=$ $1,2)$ is the density at which $E_{F}$ changes its sign, i.e., the topology of the Fermi surface changes at $n_{t}$.

where $R_{H}^{0}=-1 /\left(e n_{e}\right)$ is the Hall coefficient for $\alpha=0$ case. Equation (19) clearly shows the Hall coefficient is enhanced due to the presence of the spin-orbit coupling. In the low-density limit, $E_{F}$ is comparable to $E_{\alpha}$ and therefore pronounced effect of the spin-orbit coupling can be realized when $E_{F}<0$ as seen in Fig. 7(b). It is interesting to notice here that Hall coefficient responds to the change in the Fermi surface topology. As shown in Fig. 7 (b), it first rises sharply until $n_{t}$ where transition occurs and then starts decreasing with further increase in density. The peak in $R_{H} / R_{H}^{0}$ at the BTP is the signature of the change in the Fermi surface topology. Generally we estimate carrier concentration from the Hall coefficient measurement. It is interesting to note that for noncentrosymmetric metals we can also estimate the strength of RSOI from this measurement by noting the transition density $n_{t}$ which depends on $\alpha$.

The finite-frequency optical conductivity $\sigma_{\nu \nu^{\prime}}(\omega)$ is arising due to the transitions between the spin-split states. Within the linear response Kubo formalism, the frequency-dependent optical conductivity is given by

$$
\sigma_{\nu \nu^{\prime}}(\omega)=\frac{1}{\hbar\left(\omega+i 0^{+}\right)} \int_{0}^{\infty} d t e^{i\left(\omega+i 0^{+}\right) t}\left\langle\left[\hat{j}_{\nu}(t), \hat{j}_{\nu^{\prime}}(0)\right]\right\rangle
$$

Here $\hat{j}_{\nu}=e \hat{v}_{\nu}$ are the components of the charge current density operator,

$$
\left\langle\left[\hat{j}_{\nu}(t), \hat{j}_{\nu^{\prime}}(0)\right]\right\rangle=\sum_{\lambda} \int d^{3} k\left\langle\lambda \mathbf{k}\left|\left[\hat{j}_{\nu}(t), \hat{j}_{\nu^{\prime}}(0)\right]\right| \lambda \mathbf{k}\right\rangle f\left(E_{\mathbf{k} \lambda}\right)
$$

denotes the quantum and thermal average in the interaction picture, $\langle\mathbf{r} \mid \lambda \mathbf{k}\rangle=\psi_{\mathbf{k}}^{\lambda}(\mathbf{r})$ and $f\left(E_{\mathbf{k} \lambda}\right)$ is the FermiDirac distribution function.

After some straight forward calculation, the real part of charge optical conductivity is given by

$$
\begin{aligned}
\operatorname{Re} \sigma_{\nu \nu^{\prime}}(\omega) & =\frac{e^{2}}{8 \pi^{2} \omega} \int d^{3} k\left[f\left(E_{\mathbf{k}-}\right)-f\left(E_{\mathbf{k}+}\right)\right] v_{\nu}^{-+}(\mathbf{k}) \\
& \times v_{\nu^{\prime}}^{+-}(\mathbf{k}) \delta\left(E_{\mathbf{k}+}-E_{\mathbf{k}-}-\hbar \omega\right),
\end{aligned}
$$




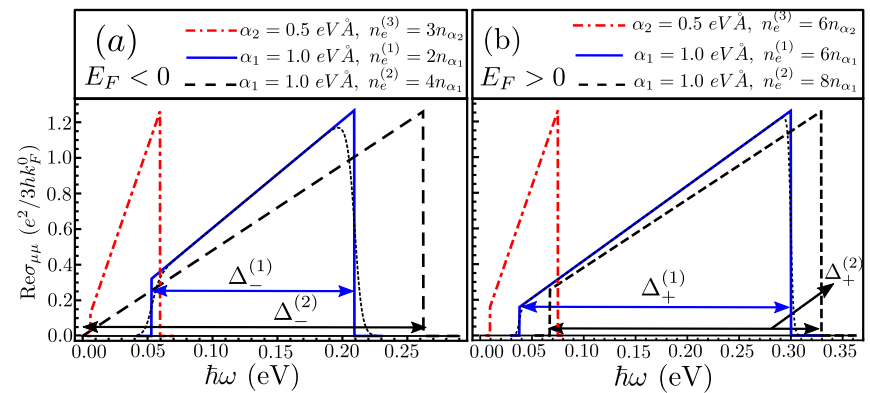

FIG. 8: (a) Plots of the Re $\sigma_{\nu \nu}$ as a function of $\hbar \omega$ for $E_{F}<0$. Solid and dashed curves corresponds to same $\alpha$ but different density and have optical absorption widths $\Delta_{1}^{-}$and $\Delta_{2}^{-}$, respectively. Here $\Delta_{-}^{(1)}<\Delta_{-}^{(2)}$ as $n_{e}^{(1)}<n_{e}^{(2)}$, so $\Delta$ depends on density as mentioned in the text. Dotted-dashed curve corresponds to different values of $\alpha$ and $n_{e}$, here $\Delta$ becomes more narrow because of the smaller value of $\alpha$. (b) Plots of the $\operatorname{Re} \sigma_{\nu \nu}$ as a function of $\hbar \omega$ for $E_{F}>0$. Solid and dashed curves corresponds to same $\alpha$ but different densities and have optical absorption widths $\Delta_{+}^{(1)}$ and $\Delta_{+}^{(2)}$, respectively. Here $\Delta_{+}^{(1)}=\Delta_{+}^{(2)}$ but $n_{e}^{(1)}<n_{e}^{(2)}$, so $\Delta$ is independent of carrier density as mentioned in the text. Dotted-dashed curve corresponds to different values of $\alpha$ and $n_{e}$, here $\Delta$ becomes more narrow because of the smaller value of $\alpha$. The dotted thin line in both the parts shows the finite temperature behavior of the optical conductivity for $\alpha_{1}$ and $n_{e}^{(1)}$.

where

$$
\begin{aligned}
v_{x}^{+-}(\mathbf{k}) & =\frac{\alpha}{\hbar}\left[e^{-2 i \phi} \sin ^{2}(\theta / 2)-\cos ^{2}(\theta / 2)\right], \\
v_{y}^{+-}(\mathbf{k}) & =\frac{i \alpha}{\hbar}\left[e^{-2 i \phi} \sin ^{2}(\theta / 2)+\cos ^{2}(\theta / 2)\right], \\
v_{z}^{+-}(\mathbf{k}) & =\frac{\alpha}{\hbar} e^{-i \phi} \sin \theta,
\end{aligned}
$$

and $v_{\nu}^{-+}(\mathbf{k})=\left[v_{\nu}^{+-}(\mathbf{k})\right]^{*}$. The only root of the equation $E_{\mathbf{k}+}-E_{\mathbf{k}-}-\hbar \omega=0$ is $k_{\omega}=\hbar \omega / 2 \alpha$. Using the result of the following angular integrations,

$$
\int_{0}^{\pi} \int_{0}^{2 \pi} v_{\nu}^{+-}(\mathbf{k}) v_{\nu^{\prime}}^{-+}(\mathbf{k}) \sin \theta d \theta d \phi=\frac{8 \pi \alpha^{2}}{3 \hbar^{2}} \delta_{\nu \nu^{\prime}}
$$

the final expression of real part of the optical conductivity at $T=0$ is given by,

$$
\begin{aligned}
\operatorname{Re} \sigma_{\nu \nu^{\prime}}(\omega) & =\frac{e^{2}}{h} \frac{k_{\omega}}{3}\left[\theta\left(2 \alpha k_{F}^{-}-\hbar \omega\right) \theta\left(\hbar \omega-2 \alpha k_{F}^{+}\right) \theta\left(E_{F}\right)\right. \\
& +\theta\left(2 \alpha k_{2}-\hbar \omega\right) \theta\left(\hbar \omega-2 \alpha k_{1}\right) \\
& \left.\times \theta\left(-E_{F}\right) \theta\left(E_{F}+E_{\alpha}\right)\right] \delta_{\nu \nu^{\prime}}
\end{aligned}
$$

Equation (25) shows the isotropic nature of the longitudinal optical conductivities: $\operatorname{Re} \sigma_{\mathrm{xx}}(\omega)=\operatorname{Re} \sigma_{\mathrm{yy}}(\omega)=$ $\operatorname{Re} \sigma_{\mathrm{zz}}(\omega) \equiv \operatorname{Re} \sigma(\omega)$ and absence of the off-diagonal conductivities: $\operatorname{Re} \sigma_{\nu \nu^{\prime}}(\omega)=0$ for $\nu \neq \nu^{\prime}$.

For $E_{F} \geq 0$, the interband optical transitions would occur when the photon energy $(\hbar \omega)$ obeys the inequality
$2 \alpha k_{+}^{F} \leq \hbar \omega \leq 2 \alpha k_{-}^{F}$ at $T=0$. However, for $E_{F} \leq 0$, the interband optical transitions take place when the photon energy satisfies the inequality $2 \alpha k_{1}^{F} \leq \hbar \omega \leq 2 \alpha k_{2}^{F}$ at $T=0$. The optical absorption width, the region where $\operatorname{Re} \sigma(\omega)$ remains nonzero, is $\Delta_{+}=8 E_{\alpha}$ for $E_{F} \geq 0$ and $\Delta_{-}=8 \sqrt{E_{\alpha}^{2}+E_{\alpha} E_{F}}$ for $E_{F} \leq 0$. Interesting to note that $\Delta_{+}$is independent of the carrier density, whereas $\Delta_{-}$depends on the both carrier density as well as Rashba energy $E_{\alpha}$ as shown in Fig. 8. The density dependence of $\Delta_{-}$is attributed to the topological change in the Fermi surface. So the optical conductivity shows a distinct response to the change in the Fermi surface topology. The absorption widths can be used to determine the value of $\alpha$ experimentally. The magnitude of the optical conductivity at the left and right edges, respectively, are $\sigma_{L / R}^{+}=\frac{e^{2} k_{-/+}^{F}}{3 \hbar}$ for $E_{F} \geq 0$ and $\sigma_{L / R}^{-}=\frac{e^{2} k_{2 / 1}^{F}}{3 \hbar}$ for $E_{F} \leq 0$. We observe that $\sigma_{L}^{+}<\sigma_{R}^{+}$and $\sigma_{L}^{-}<\sigma_{R}^{-}$which is also clear from Fig. 8.

It is interesting to note that the spin-split energy gap is of the order of $0.1 \mathrm{eV}$ for the carrier density $n_{e}=10^{25}$ $\mathrm{m}^{-3}$ and $\alpha=1.0 \times 10^{-10} \mathrm{eV}-\mathrm{m}$. This energy scale is comparable to the electromagnetic radiation with very high frequency $\omega \sim 10^{14} \mathrm{~Hz}$. The high-frequency radiation would flip the spin in a very short time. The noncentrosymmetric semiconductors can be used for highspeed spintronic devices. It should be emphasized here that the spin-orbit coupling locks electron's spin with its momentum which changes due to scattering from impurities. Hence the charge carrier's spin can flip due to strong spin-orbit coupling. Typically, the spin scattering rate for Elliot-Yafet and Dykonov-Perev mechanisms $\operatorname{are}^{65} \tau / \tau_{s}^{E Y} \sim\left(\Delta_{\mathrm{so}} / E_{F}\right)^{2}$ and $1 / \tau_{s}^{D P} \sim \tau E_{\alpha} E_{F} / \hbar^{2}$, respectively. Here $\Delta_{\text {so }}=\alpha k_{F}$ and $\tau$ being the momentum scattering time. Therefore, the spin-flip scattering rate can be reduced for suitable choice of moderate density and weak spin-orbit coupling, which will be the criteria for this system to use it for good spintronic device applications at low temperature.

\section{SUMMARY AND CONCLUSIONS}

In summary, we have theoretically studied signatures of the Fermi surface topology change in thermoelectric and optical properties of noncentrosymmetric metals. The noncentrosymmetric metals possess distinct Fermi surface topology which depends on the sign of the Fermi energy. As a result of this, the chemical potential is found to exhibit a dimensional crossover from 3D to 1D-like behavior as the Fermi energy switches its sign from positive to negative one. It is shown that the electrical conductivity is continuously differentiable at the band touching point, as opposed to 2D Rashba systems. There is a significant enhancement of thermopower in the low density regime which is responsible to obtain a remarkable thermoelectric figure of merit with value more than 2 . However the figure of merit is found to decrease with 
the increase of the strength of the Rashba spin-orbit interaction. This feature is explained qualitatively. It is shown that the Hall coefficient first rises sharply until BTP and then starts decreasing with further increase in density. The absorption width above the BTP depends solely on the spin-orbit coupling strength. Hence Hall coeffiecient and optical conductivity measurements can be used to extract $\alpha$ experimentally. However, the absorption width below the BTP depends on both the density and $\alpha$. The spin-split energy gap is comparable to the electromagnetic radiation with high frequency $\omega \sim 10-100 \mathrm{THz}$. The corresponding spin-flip time scale will be very small. Therefore, noncentrosymmetric bulk materials can be used for good thermoelectric as well as spintronics devices with appropriate system parameters at low temperatures.

\section{ACKNOWLEDGEMENT}

T.B. sincerely acknowledges the financial support provided by the University of North Bengal to pursue this work. We also acknowledge Dr. Arijit Kundu for some useful discussions.

\section{Appendix A}

\section{Calculation of the relaxation time}

Relaxation time approximation for multiband systems: In this Appendix, we present calculation of the relaxation time by solving the Boltzmann transport equation including the interband scattering for $E>0$ and interbranch scattering for $E<0$ self-consistently. We consider electrons in noncentrosymmetric semiconductors with spin-independent short-range scatterer. This system is subjected to a spatially uniform electric field $\boldsymbol{\Xi}$ and temperature gradient $\boldsymbol{\nabla} T$. The effective electric field due to charge redistribution results from $\boldsymbol{\Xi}$ is given by $\boldsymbol{\Xi}_{\text {eff }}=$ $\boldsymbol{\Xi}-(\boldsymbol{\nabla} \mu) / e$. We now linearize the Fermi-Dirac distribution function $f_{\xi}\left(E_{\xi}\right)=f_{\xi}^{0}\left(E_{\xi}\right)+\delta f_{\xi}$ around the equilibrium solution $f_{\xi}^{0}\left(E_{\xi}\right)$. Here $\xi \equiv(\lambda, \mathbf{k})$ for $E \geq 0$ and $\xi \equiv(\eta, \mathbf{k})$ for $-E_{\alpha} \leq E \leq 0$ is the eigenstate index; and $\delta f_{\xi}$ is the out-of-equilibrium deviation which is linear in the external electric field. In nonequilibrium steady states, the linearized Boltzmann transport equation ${ }^{66}$ for the charge carriers is

$$
\mathbf{F}_{\xi} \cdot \mathbf{v}_{\xi} \frac{\partial f^{0}}{\partial E_{\xi}}=-\sum_{\xi^{\prime}} W_{\xi^{\prime}, \xi}\left(\delta f_{\xi}-\delta f_{\xi^{\prime}}\right) .
$$

Here the generalized force acting on the state $\xi$ is

$$
\mathbf{F}_{\xi}=-\frac{\left(E_{\xi}-\mu\right)}{T} \nabla T+e \boldsymbol{\Xi}_{\mathrm{eff}},
$$

and $\mathbf{v}_{\xi}$ is the group velocity of the state $\xi$. Also, $W_{\xi^{\prime}, \xi}$ is the transition rate from the state $\xi$ to the state $\xi^{\prime}$. We have used the fact that $W_{\xi^{\prime}, \xi}=W_{\xi, \xi^{\prime}}$ in the Boltzmann transport equation. Within the lowest-order Born approximation, the intra-band transition rate between the states $\xi$ and $\xi^{\prime}$ is

$$
W_{\xi^{\prime} \xi}=\frac{2 \pi N_{\mathrm{imp}}}{\hbar}\left|\left\langle\phi_{\xi^{\prime}}\left(\mathbf{k}^{\prime}\right)|U(\mathbf{r})| \phi_{\xi}(\mathbf{k})\right\rangle\right|^{2} \delta\left(E_{\xi}-E_{\xi^{\prime}}\right) .
$$

Here, $N_{\mathrm{imp}}$ is the number of $\delta$-scatterer randomly distributed in the system at various locations $\mathbf{r}_{i}$. The corresponding spin-independent impurity potential produced by the $\delta$-scatterer is given by $U(\mathbf{r})=U_{0} \sum_{i=1}^{N_{\text {imp }}} \delta\left(\mathbf{r}-\mathbf{r}_{i}\right)$. Here $U_{0}$ being the strength of the impurity potential, whose dimension is energy times volume. The Fourier transform of the potential $U(\mathbf{r})$ is $U(\mathbf{q})=U_{0}$. Upon simplification, the transition rates are obtained as

$$
\begin{aligned}
& W_{\lambda^{\prime} \lambda}=\frac{1}{u_{0} V}\left(1+\lambda \lambda^{\prime} \cos \theta\right) \delta\left(E_{\lambda}-E_{\lambda^{\prime}}\right) ; \quad E \geq 0, \\
& W_{\eta^{\prime} \eta}=\frac{1}{u_{0} V}(1+\cos \theta) \delta\left(E_{\eta}-E_{\eta^{\prime}}\right) ; \quad-E_{\alpha} \leq E \leq 0,
\end{aligned}
$$

where $1 / u_{0}=\pi n_{\text {imp }} U_{0}^{2} / \hbar$ with $n_{\text {imp }}$ being the impurity density.

We need to solve the Boltzmann transport equation separately for $E \geq 0$ and $-E_{\alpha} \leq E \leq 0$. This is because the intraband and interband transitions take place when $E \geq 0$. Whereas intrabranch and interbranch transition occurs within the band $\lambda=-$. We are able to obtain exact analytical expression of the scattering time even if we 
keep the interband/interbranch contribution in the Boltzmann transport equation. Assuming the out-of-equilibrium distribution function is of the following form:

$$
\delta f_{\xi}\left(E, \mathbf{v}\left(E_{\xi}, \theta, \phi\right)\right)=-\frac{\partial f^{0}(E)}{\partial E} \mathbf{F}_{\xi} \cdot \mathbf{v}_{\xi}(E, \theta, \phi) \tau_{\xi}(E)
$$

Substituting Eq. (A5) into Eq. (A1), the self-consistent equation for the relaxation time $\tau_{\xi}(E)$ is

$$
\frac{1}{\tau_{\xi}(E)}=\frac{V}{4 \pi} \sum_{\xi^{\prime}} \int d E_{\xi^{\prime}} D_{\xi^{\prime}}\left(E_{\xi^{\prime}}\right) \sin \theta^{\prime} d \theta^{\prime} d \phi^{\prime} W_{\xi \xi^{\prime}}\left(E_{\xi}, E_{\xi^{\prime}}\right)\left[1-\frac{\mathbf{F}_{E_{\xi^{\prime}}} \cdot \mathbf{v}\left(E_{\xi^{\prime}}, \theta^{\prime}, \phi^{\prime}\right)}{\mathbf{F}_{E_{\xi}} \cdot \mathbf{v}\left(E_{\xi}, \theta, \phi\right)} \frac{\tau_{\xi^{\prime}}\left(E_{\xi^{\prime}}\right)}{\tau_{\xi}\left(E_{\xi}\right)}\right] .
$$

First we consider $E \geq 0$ case. After some straightforward calculation, we get the following self-consistent equation for the relaxation time $\tau_{\lambda}(E)$ :

$$
\frac{1}{\tau_{\lambda}(E)}=\sum_{\lambda^{\prime}} \frac{D_{\lambda^{\prime}}(E)}{2 u_{0}} \int d \theta^{\prime} \sin \theta^{\prime}\left(1+\lambda \lambda^{\prime} \cos \theta^{\prime}\right)\left[1-\cos \theta^{\prime} \frac{\tau_{\lambda^{\prime}}(E)}{\tau_{\lambda}(E)}\right]
$$

Performing the integrals and summation, the above equation reduces to

$$
\frac{1}{\tau_{ \pm}(E)}=\frac{2 D_{ \pm}(E)}{3 u_{0}}+\frac{D_{\mp}(E)}{u_{0}}\left[1+\frac{\tau_{\mp}(E)}{3 \tau_{ \pm}(E)}\right] .
$$

On solving the above coupled algebraic equations, the relaxation times of the two bands for $E \geq 0$ are obtained as

$$
\tau_{\lambda}(E)=u_{0}\left[\frac{D_{\lambda}(E)}{\left(D_{T}^{>}(E)\right)^{2}}+\frac{1}{2 D_{T}^{>}(E)}\right] .
$$

For $-E_{\alpha}<E<0$, the self-consistent equations for the relaxation time $\tau_{\eta}(E)$ are

$$
\frac{1}{\tau_{\eta}(E)}=\sum_{\eta^{\prime}} \frac{D_{\eta^{\prime}}(E)}{2 u_{0}} \int d \theta^{\prime} \sin \theta^{\prime}\left(1+\cos \theta^{\prime}\right)\left[1-(-1)^{\eta^{\prime}-\eta} \cos \theta^{\prime} \frac{\tau_{\eta^{\prime}}(E)}{\tau_{\eta}(E)}\right] .
$$

The solutions for the relaxation times are obtained as

$$
\tau_{\eta}(E)=u_{0}\left[\frac{D_{\eta}(E)}{\left(D_{T}^{<}(E)\right)^{2}}+\frac{1}{2 D_{T}^{<}(E)}\right] .
$$

In our case, total density of states for $E \geq 0$ and $E<0$ have the same form,

$$
D_{T}^{>}(E) \equiv D_{T}^{<}(E)=\frac{1}{2 \pi^{2}}\left(\frac{2 m^{*}}{\hbar^{2}}\right)^{\frac{3}{2}}\left[\frac{E+2 E_{\alpha}}{\sqrt{E+E_{\alpha}}}\right] .
$$

As a consequence of the same energy dependence of the total density of states below and above the BTP, we also have the same form of the relaxation time for $E \geq 0$,

$$
\tau_{\lambda}(E)=2 \pi^{2} u_{0}\left(\frac{\hbar^{2}}{2 m^{*}}\right)^{\frac{3}{2}}\left(\frac{\sqrt{E+E_{\alpha}}}{E+2 E_{\alpha}}\right)\left[1-\lambda \frac{\sqrt{4 E_{\alpha}\left(E+E_{\alpha}\right)}}{\left(E+2 E_{\alpha}\right)}\right]
$$

and for $E<0$,

$$
\tau_{\eta}(E)=2 \pi^{2} u_{0}\left(\frac{\hbar^{2}}{2 m^{*}}\right)^{\frac{3}{2}}\left(\frac{\sqrt{E+E_{\alpha}}}{E+2 E_{\alpha}}\right)\left[1-(-1)^{\eta-1} \frac{\sqrt{4 E_{\alpha}\left(E+E_{\alpha}\right)}}{\left(E+2 E_{\alpha}\right)}\right]
$$

\section{The electrical conductivity at $T=0$}

Within the semiclassical Boltzmann transport theory ${ }^{55}$, the general expression of the electrical conductivity at $T=0$ for $E \geq 0$ is given by

$$
\sigma_{\nu \nu}^{>}=\frac{e^{2}}{4 \pi} \sum_{\lambda= \pm 1} \int_{0}^{\infty} d E\left(-\partial_{E} f^{0}(E)\right) \int_{0}^{\pi} \int_{0}^{2 \pi} \sin \theta d \theta d \phi D_{\lambda}(E)\left\langle\hat{v}_{\nu}(E, \theta, \phi)\right\rangle_{\lambda}^{2} \tau_{\lambda}(E)
$$


where $\nu=x, y, z$ and $\hat{v}_{\nu}(E, \theta, \phi)$ is the $\nu$-component of the velocity operator and $\tau_{\lambda}(E)$ is the relaxation time. The expectation values of the velocity operator $\hat{v}_{\mu}$ with respect to the $\lambda= \pm 1$ states are $\left\langle\hat{v}_{x}(E, \theta, \phi)\right\rangle_{\lambda}=\left(\frac{\hbar k_{\lambda}(E)}{m^{*}}+\right.$ $\left.\frac{\lambda \alpha}{\hbar}\right) \sin \theta \cos \phi$ and so on. As we have already seen that $\tau_{\lambda}(E)$ is independent of angular variables, so using

$$
\int_{0}^{\pi} \int_{0}^{2 \pi}\left\langle\hat{v}_{\nu}(E, \theta, \phi)\right\rangle_{\lambda}^{2} \sin \theta d \theta d \phi=\frac{4 \pi}{3}\left(\frac{\hbar k_{\lambda}(E)}{m^{*}}+\lambda \frac{\alpha}{\hbar}\right)^{2},
$$

which exhibits isotropic nature of the electrical conductivity: $\sigma_{x x}=\sigma_{y y}=\sigma_{z z}=\sigma$. Using the forms of density of states and Eq. (A13), we get

$$
\sigma^{>}=\frac{e^{2}}{h} \frac{2 \hbar^{2}}{m^{*} n_{\mathrm{imp}} U_{0}^{2}}\left[1-\frac{E_{F}^{2}}{3\left(2 E_{\alpha}+E_{F}\right)^{2}}\right]\left(E_{\alpha}+E_{F}\right) .
$$

Similarly for $E<0$, the electrical conductivity becomes

$$
\begin{aligned}
\sigma^{<} & =\frac{e^{2}}{4 \pi} \sum_{\eta=1,2} \int_{-E_{\alpha}}^{0} d E\left(-\partial_{E} f^{0}(E)\right) \int_{0}^{\pi} \int_{0}^{2 \pi} \sin \theta d \theta d \phi D_{\eta}(E)\left\langle\hat{v}_{\nu}(E, \theta, \phi)\right\rangle_{\eta}^{2} \tau_{\eta}(E), \\
& =\frac{e^{2}}{h} \frac{2 \hbar^{2}}{m^{*} n_{\mathrm{imp}} U_{0}^{2}}\left[1-\frac{E_{F}^{2}}{3\left(2 E_{\alpha}+E_{F}\right)^{2}}\right]\left(E_{\alpha}+E_{F}\right) .
\end{aligned}
$$

It is clear from Eqs. (A17) and (A18) that electrical conductivity has same form below $\left(E_{F}<0\right)$ and above $E_{F} \geq 0$ the BTP. It is also clear from Fig. 3 that electrical conductivity has the same kind of energy dependence below and above the BTP. So in our case electrical conductivity will be continuously differentiable at the BTP.

1 S. A. Wolf, D. D. Awschalom, R. A. Buhrman, J. M. Daughton, S. von. Molnar, M. L. Roukes, A. Y. Chtchelkanova, and D. M Treger, Science 294, 1488 (2001).

2 R. Winkler, Spin-Orbit Coupling Effets in Twodimensional Electron and Hole Systems (Springer Berlin Heidelberg, 2003).

3 I. Zutic, J. Fabian, and S. Das Sarma, Rev. Mod. Phys. 76, 323 (2004)

4 E. I. Rashba, Sov. Phys. Solid State 2, 1109 (1960).

5 Y. A. Bychkov and E. I. Rashba, J. Phys. C 17, 6039 (1984).

${ }^{6}$ G. Dresselhaus, Phys. Rev. 100, 580 (1955).

7 J. Nitta, T. Akazaki, H. Takayanagi, and T. Enoki, Phys. Rev. Lett. 78, 1335 (1997).

8 S. Murakami, N. Nagaosa, and S. C. Zhang, Science 301, 1348 (2003)

9 S. Murakami, N. Nagaosa, and S. C. Zhang, Phys. Rev. B 69, 235206 (2004)

10 J. E. Hirsch, Phys. Rev. Lett. 83, 1834 (1999).

11 S. Zhang, Phys. Rev. Lett. 85, 393 (2000).

12 J. Sinova, D. Culcer, Q. Niu, N. A. Sinitsyn, T. Jungwirth, and A. H. MacDonals, Phys. Rev. Lett. 92, 126603 (2004).

13 Y. K. Kato, R. C. Myers, A. C. Gossard, and D. D. Awschalom, Science 306, 1910 (2004)

14 B. A. Bernevig and S. C. Zhang, Phys. Rev. Lett. 96, 106802 (2006).

15 J. Sinova, S. O. Valenzuela, J. Wunderlich, C. H. Back, and T. Jungwirth, Rev. Mod. Phys. 87, 1213 (2015).

16 K. Tsutsui and S. Murakami, Phys. Rev. B 86, 115201 (2012).
17 S. D. Ganichev, E. L. Ivchenko, V. V. Belkov, S. A. Tarasenko, M. Sollinger, D. Weiss, W. Wegscheider, and W. Prettl, Nature 417, 153 (2002).

18 C. R. Ast, J. Henk, A. Ernst, L. Moreschini, M. C. Falub, D. Pacile, P. Bruno, K. Kern, and M. Grioni, Phys. Rev. Lett. 98, 186807 (2007).

19 M. Bianchi, D. Guan, S. Bao, J. Mi, B. B. Iversen, P. D.C. King, and P. Hofmann, Nature Communications 1, 128 (2010).

20 P. D. C. King, R. C. Hatch, M. Bianchi, R. Ovsyannikov, C. Lupulescu, G. Landolt, B. Slomski, J. H. Dil, D. Guan, J. L. Mi, E. D. L. Rienks, J. Fink, A. Lindblad, S. Svensson, S. Bao, G. Balakrishnan, B. B. Iversen, J. Osterwalder, W. Eberhardt, F. Baumberger, and Ph. Hofmann, Phys. Rev. Lett. 107, 096802 (2011).

${ }^{21}$ K. Ishizaka, M. S. Bahramy, H. Murakawa, M. Sakano, T. Shimojima, T. Sonobe, K. Koizumi, S. Shin, H. Miyahara, A. Kimura, K. Miyamoto, T. Okuda, H. Namatame, M. Taniguchi, R. Arita, N. Nagaosa, K. Kobayashi, Y. Murakami, R. Kumai, Y. Kaneko, Y. Onose, and Y. Tokura, Nat. Mater. 10, 521 (2011).

22 M. S. Bahramy, R. Arita, and N. Nagaosa, Phys. Rev. B 84, 041202(R) (2011).

23 S. V. Eremeev, I. A. Nechaev, Yu. M. Koroteev, P. M. Echenique, and E. V. Chulkov, Phys. Rev. Lett. 108, 246802 (2012).

${ }^{24}$ G. Landolt, S. V. Eremeev, Y. M. Koroteev, B. Slomski, S. Muff, T. Neupert, M. Kobayashi, V. N. Strocov, T. Schmitt, Z. S. Aliev, M. B. Babanly, I. R. Amiraslanov, E. V. Chulkov, J. Osterwalder, and J. H. Dil, Phys. Rev. 
Lett. 109, 116403 (2012).

25 M. Sakano, M. S. Bahramy, A. Katayama, T. Shimojima, H. Murakawa, Y. Kaneko, W. Malaeb, S. Shin, K. Ono, H. Kumigashira, R. Arita, N. Nagaosa, H. Y. Hwang, Y. Tokura, and K. Ishizaka, Phys. Rev. Lett. 110, 107204 (2013).

26 J. Kang and J. Zang, Phys. Rev. B 91, 134401 (2015).

27 V. P. Mineev and Y. Yoshioka, Phys. Rev. B 81, 094525 (2010).

${ }^{28}$ K. Togano, P. Badica, Y. Nakamori, S. Orimo, H. Takeya, and K. Hirata, Phys. Rev. Lett. 93, 247004 (2004).

29 M. Smidman, M. B. Salamon, H. Q. Yuan, and D. F. Agterber, Rep. Prog. Phys. 80, 036501 (2017).

30 M. Uchida, Y. Onose, Y. Matsui, and Y. Tokura, Science 311, 359 (2006).

31 U. K. Roszler, A. N. Bogdanov, and C. Pfleiderer, Nature 442, 797 (2006).

32 S. Muhlbauer, B. Binz, F. Jonietz, C. Pfleiderer, A. Rosch, A. Neubauer, R. Georgii, and P. Böni, Science 323, 915 (2009).

33 X. Z. Yu, Y. Onose, N. Kanazawa, J. H. Park, J. H. Han, Y. Matsui, N. Nagaosa, and Y. Tokura, Nature 465, 901 (2010).

34 K. V. Samokhin, Phys. Rev. B 78, 144511 (2008).

35 V. P. Mineev, Phys. Rev. B 88, 134514 (2013).

${ }^{36}$ K. V. Samokhin, Phys. Rev. B 76, 094516 (2007).

37 S. X. Wang, H. R. Chang, and J. Zhou, Phys. Rev. B 96, 115204 (2017).

38 H. Murakawa, M. S. Bahramy, M. Tokunaga, Y. Kohama, C. Bell, Y. Kaneko, N. Nagaosa, H. Y. Hwang, and Y. Tokura, Science 342, 1490 (2013).

39 C. Bell, M. S. Bahramy, H. Murakawa, J. G. Checkelsky, R. Arita, Y. Kaneko, Y. Onose, M. Tokunaga, Y. Kohama, N. Nagaosa, Y. Tokura, and H. Y. Hwang, Phys. Rev. B 87, 081109(R) (2013).

${ }^{40}$ C. Martin, E. D. Mun, H. Berger, V. S. Zapf, and D. B. Tanner, Phys. Rev. B 87, 041104(R) (2013).

41 T. Ideue, J. G. Checkelsky, M. S. Bahramy, H. Murakawa, Y. Kaneko, N. Nagaosa, and Y. Tokura, Phys. Rev. B 90, 161107(R) (2014).

42 L. Ye, J. G. Checkelsky, F. Kagawa, and Y. Tokura, Phys. Rev. B 91, 201104(R) (2015).

43 C. R. Wang, J. C. Tung, R. Sankar, C. T. Hsieh, Y. Y. Chien, G. Y. Guo, F. C. Chou, and W. L. Lee, Phys. Rev. B 88, 081104(R) (2013).

44 V. Brosco and C. Grimaldi, Phys. Rev. B 95, 195164 (2017).

45 L. Wu, J. Yang, S. Wang, P. Wei, J. Yang, W. Zhang, and
L. Chen, Phys. Rev. B 90, 195210 (2014).

46 T. Ideue, L. Ye, J. G. Checkelsky, H. Murakawa, Y. Kaneko, and Y. Tokura, Phys. Rev. B 92, 115144 (2015).

47 N. Ogawa, M. S. Bahramy, H. Murakawa, Y. Kaneko, and Y. Tokura, Phys. Rev. B 88, 035130 (2013).

48 S. Maiti, V. Zyuzin, and D. L. Maslov, Phys. Rev. B 91, 035106 (2015).

49 P. Di Pietro, F. M. Vitucci, D. Nicoletti, L. Baldassarre, P. Calvani, R. Cava, Y. S. Hor, U. Schade, and S. Lupi, Phys. Rev. B 86, 045439 (2012).

50 I. I. Boiko and E. I. Rashba, Fiz. Tverd. Tela (Leningrad) 2, 1874 (1960) [Sov. Phys. Solid State 2, 1692 (1960)].

51 G. A. H. Schober, H. Murakawa, M. S. Bahramy, R. Arita, Y. Kaneko, Y. Tokura, and N. Nagaosa, Phys. Rev. Lett. 108, 247208 (2012).

52 M. A. Chernikov, L. Degiorgi, E. Felder, S. Paschen, A. D. Bianchi, H. R. Ott, J. L. Sarrao, Z. Fisk, and D. Mandrus, Phys. Rev. B 56, 1366 (1997).

53 J. F. DiTusa, S. B. Zhang, K. Yamaura, Y. Xiong, J. C. Prestigiacomo, B. W. Fulfer, P. W. Adams, M. I. Brickson, D. A. Browne, C. Capan, Z. Fisk, and Julia Y. Chan, Phys. Rev. B 90, 144404 (2014).

54 K.-W. Lee and W. E. Pickett, Phys. Rev. B 72, 174505 (2005).

55 N. W. Ashcroft and N. D. Mermin, Solid State Physics (Holt, Rinehart and Winston, 1976).

56 C. Xiao, D. Li, and Z. Ma, Phys. Rev. B 93, 075150 (2016).

57 L. D. Hicks and M. S. Dresselhaus, Phys. Rev. B 47, 12727 (1993).

58 L. D. Hicks and M. S. Dresselhaus, Phys. Rev. B 47, 16631 (1993).

59 Valentina Brosco, Lara Benfatto, Emmanuele Cappelluti, and Claudio Grimaldi, Phys. Rev. Lett. 116, 166602 (2016).

60 G. D. Mahan, Many-Particle Physics (Springer, Berlin, 2000).

61 A. Mawrie and T. K. Ghosh, J. of Appl. Phys. 119, 044303 (2016).

62 X. Zotos, F. Naef, M. Long, and P. Prelovsek, Phys. Rev. Lett. 85, 377 (2000).

63 P. Prelovsek and X. Zotos, Phys. Rev. B 64, 235114 (2001).

64 T. Stauber, N. M. R. Peres, and A. K. Geim, Phys. Rev. B 78, 085432 (2008).

65 E. Y. Tsymbal and I. Zutic, Handbook of Spin Transport and Magnetism (CRC press, 2011).

66 J. M. Ziman, Principles of the Theory of Solids, (Cambridge University Press, Cambridge, MA, 1972). 